\title{
Batch Measurement of Attenuation Length of Wavelength-shifting Fibers for LHAASO Electromagnetic Detectors
}

\section{Xiaopeng Zhang}

Institute of High Energy Physics, CAS

E-mail: zhangxpeihep.ac.cn

\section{Chao Hou, Xiangdong Sheng, for the LHAASO collaboration}

Institute of High Energy Physics, CAS

Over $700 \mathrm{~km}$ of wavelength-shifting fibers are being used in 5242 electromagnetic detectors (EDs) of the LHAASO project. It is important to monitor the attenuation length of the fibers among spools for quality control as well as the performance uniformity among detectors. An apparatus was built-up in the laboratory of IHEP for the purpose. Eight fibers with length of $5.4 \mathrm{~m}$ could be measured in one round of testing within 15 minutes with the current configuration and will be upgraded to 16 after a further upgrade. The measurement has a systematic uncertainty of less than $3 \%$ and meets the requirement of the application.

36th International Cosmic Ray Conference -ICRC2019-

July 24th - August 1st, 2019

Madison, WI, U.S.A. 


\section{Introduction}

\subsection{LHAASO experiment and ED}

The Large High Altitude Air Shower Observatory (LHAASO) [1] is a hybrid cosmic ray observatory under construction at an altitude of $4410 \mathrm{~m}$ a.s.l. in Sichuan Provence, China. LHAASO is designed to discover high energy gamma ray sources, and study the origin, acceleration, and propagation of various cosmic ray species by measuring the energy spectrum, elemental composition and anisotropy with high resolution.

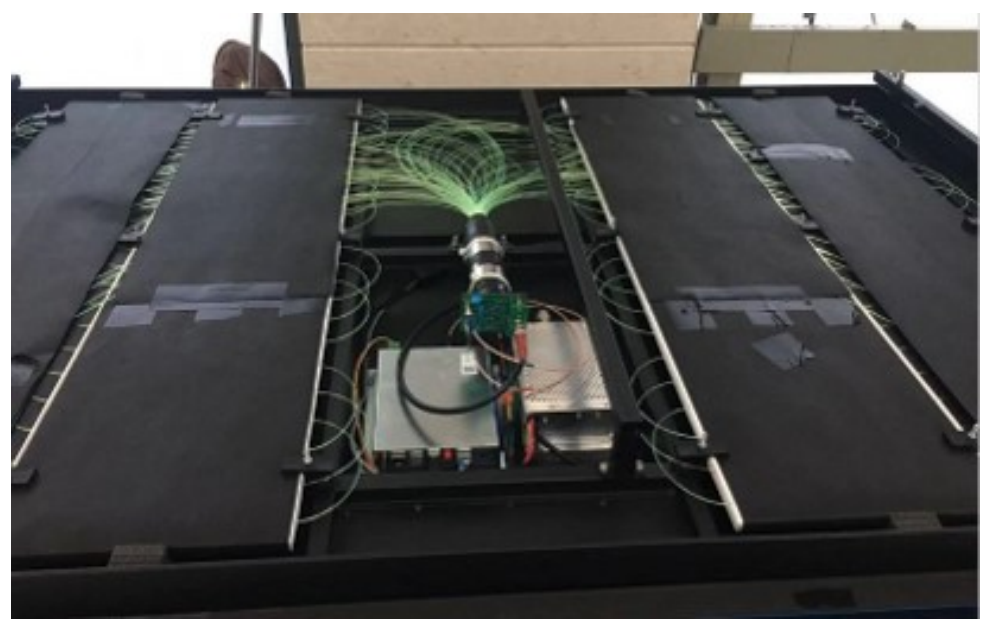

Figure 1: Photograph of the inner structure of an ED

As one of the four types of LHAASO detectors [2], electromagnetic detector (ED) array consists of 5242 scintillation detectors and covers an area of about $1.3 \mathrm{~km}^{2}$. The goal of the ED array is to measure the densities and arrival times of secondary particles in extensive air showers (EASs) [3]. As shown in Figure 1, four identical sensitive units are housed in a light insulated steel shell, each includes a BC-408 plastic scintillator with a dimension of $100 \times 25 \times 1 \mathrm{~cm}^{3} .24$ grooves are machined on one surface of the scintillator for 12 BCF-92 wavelength shifting (WLS) fibers passing through. All 96 fiber ends are bunched together and polished, then coupled onto the window of an XP3960 photomultiplier tube (PMT). The PMT is powered by a high-voltage supply. The charge and time information of its output signals are digitalized by customized circuits, then transferred to the data acquisition system (DAQ) via the White Rabbit (WR) network.

\subsection{Wavelength-shifting fiber}

WLS fibers play a major role in the ED to collect scintillation photons. The UV to violet light (350-450 nm) emitted by the plastic scintillator is absorbed by a fluorescent dye of the WSL fiber, then the dye emits green (475-575 nm) light. The absorption and emission spectrum of BCF-92 fiber are shown in Figure 2(a). The response function of XP3960 PMT (Figure 2(b)) is chosen to match the emission spectrum.

In order to achieve a higher light output, the attenuation of the emitted light inside the WLS fiber should be as low as possible. Light attenuation in a uniform medium follows the exponential 


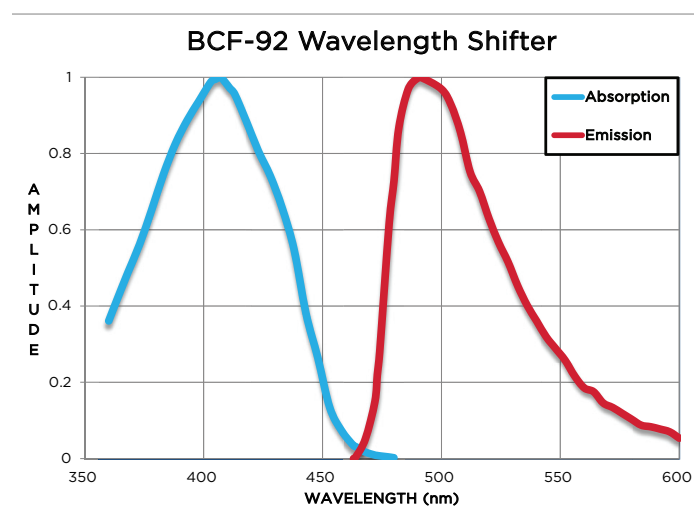

(a)

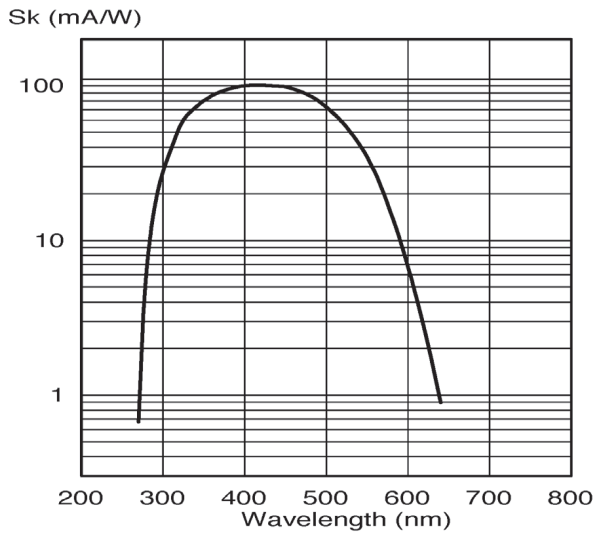

(b)

Figure 2: (a) The absorption and emission spectra of BCF-92 WLS fiber. (b) The wavelength response of XP3960 PMT.

low, i.e.:

$$
I(x)=I_{0} \exp (-x / \lambda),
$$

where $I_{0}$ is the input light intensity at the original point and $I(x)$ is the light intensity measured at a distance of $x$. The parameter $\lambda$ is the attenuation length.

All the 11 batches of WLS fibers utilized by LHAASO-ED will be manufactured and delivered by Saint-Gobain Crystals in about two years, about $720 \mathrm{~km}$ in total length. Monitoring the attenuation length is important since fibers with lower attenuation length result in poor performance on the number of photoelectrons (Npe). Moreover, the fluctuation of attenuation length with spools and batches will have an influence on the uniformity between EDs.

To experimentally measure the attenuation length of a particular fiber, the basic idea is to excite the fiber at different positions and measure the light outputs at the fixed fiber end. Then the attenuation length can be obtained by exponentially fitting the $I-x$ curve.

\section{Experimental Setup}

\subsection{Measurement system}

A semi-automatic testing bench has been built in IHEP laboratory to perform the attenuation length measurement with high efficiency.

The schematic of the system is shown in Figure 3(a), which consists of following parts:

1. Mechanical part

A linear rail of $3 \mathrm{~m}$ length is placed in a dark box. A carriage carrying the light source can move along the rail, driven by a servo motor. At both ends of the rail, 8 pairs of magnetic fiber fixtures are installed (shown in Figure 3(b)). WSL fibers under test are fixed by these fixtures and slightly tensioned.

2. Optical part

A block made of black polyethylene was mounted on the carriage. Each fiber passes through 

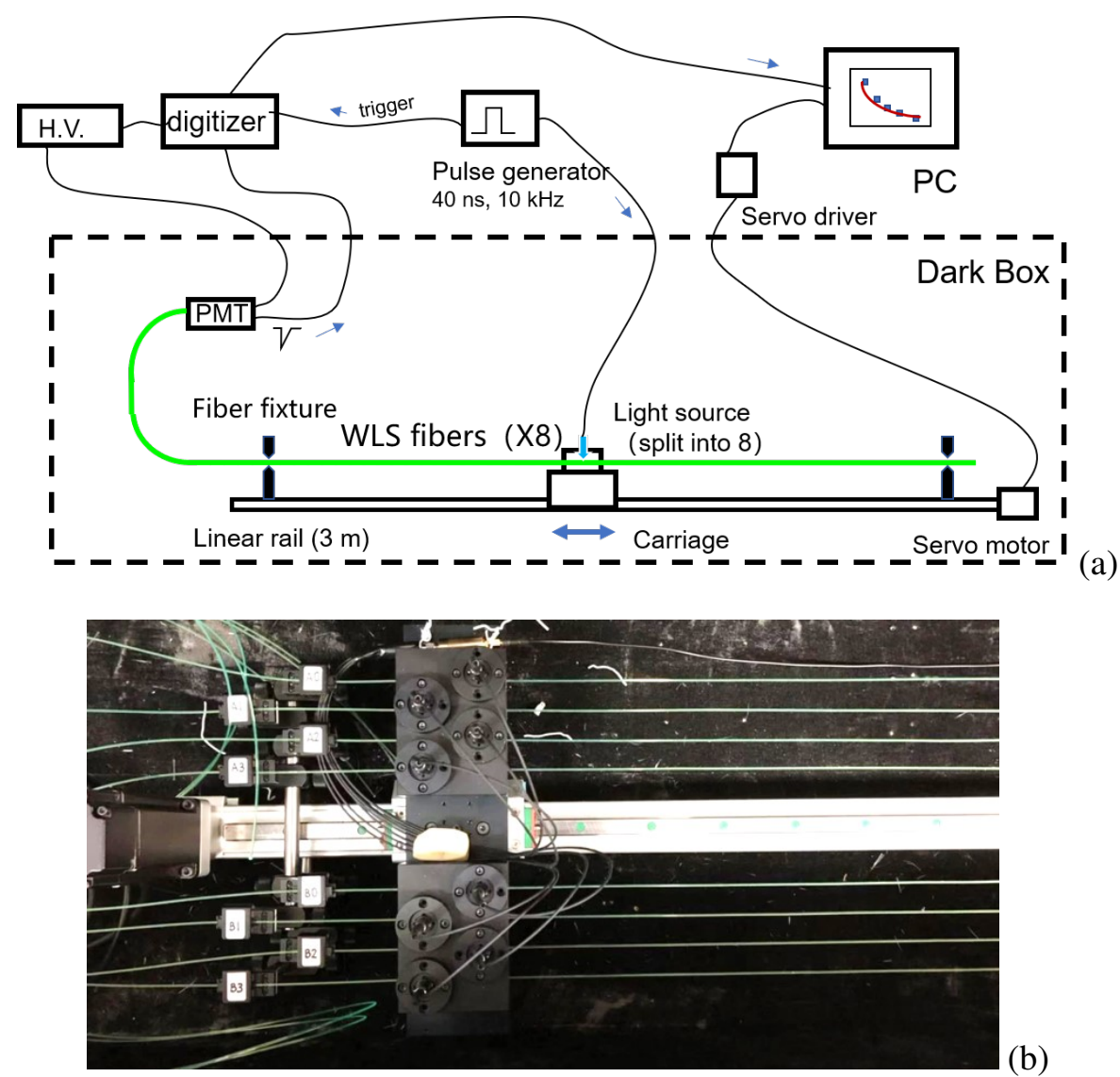

Figure 3: (a) Schematic of the fiber testing system. (b) Photograph shows one end of the rail, carriage, light source, and fiber fixtures.

one of the 8 horizontal holes in the block. The light source is a $420 \mathrm{~nm}$ LED, driven by a pulse generator, emits light pulses with a width of $30 \mathrm{~ns}$ and a repetition rate of $10 \mathrm{kHz}$. The LED is coupled to an optical fiber and split into 8 fibers by a Y-shaped optical splitter. Each optical fiber terminates at the top of the block with a beam expanding lens. Collimated UVlight irradiates through vertical holes and excites the WSL fibers. The ends of WLS fibers are coupled to XP3960 photomultiplier tubes (PMTs).

3. Electronic part

Electronic boards and high-voltage power units are the same as we used in the LHAASO-ED detectors. The electronic boards are externally triggered by the pulse generator. The output signals of PMTs are time-integrated. Charge and timestamp are sent to a WR switch and finally to the PC.

\section{Software}

A computer program with graphic user interface (GUI) is developed to fulfill the testing procedure (Figure 4). The software includes driver to control the servo motor and DAQ interface. In a general practice, we measure 30 points for every WSL fiber. At each position the data acquisition lasts $20 \mathrm{sec}$ then the motor starts and brings the carriage to the next 


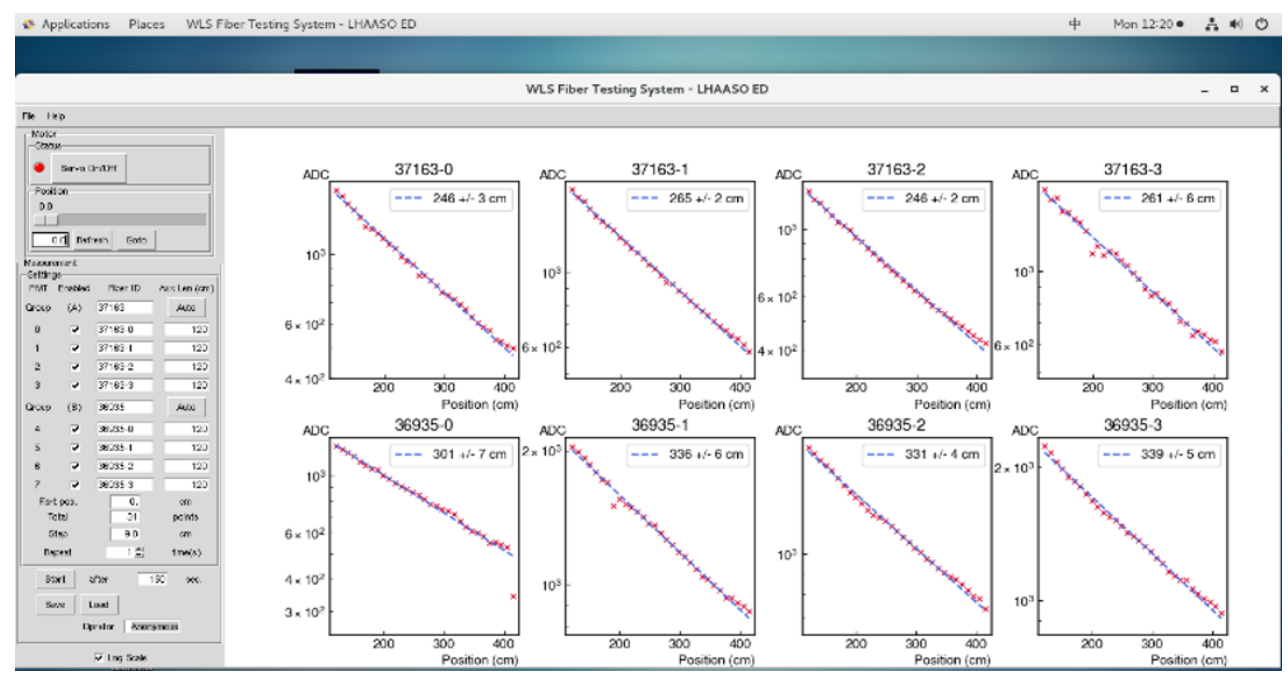

Figure 4: Screenshot of the software during measurement.

position. The number of total points can be set in the software interface as well as the stepping distance. Data points are visualized on the plots during the measurement. When the measurement is completed, it applies an exponential fitting (Eq. 1.1) to all curves and saves results to local disk.

\subsection{Performance}

The positioning accuracy of the carriage on the rail is better than $0.1 \mathrm{~mm}$. With a setting of acquiring data at one position for $20 \mathrm{~s}$, the light yield can be measured with a resolution of $<1 \%$. A typical overall uncertainty of the attenuation length results is $\sim 2 \%$. The fibers to be tested can be easily installed or replaced in several minutes. It takes about 15-20 min to finish all the 30 points (8 fibers).

To evaluate the stability of the system, we performed a series of repetitive measurements, in which 8 fibers were independently measured over 30 times. Between each measurments the dark box is reopen and fibers are detached and reinstalled. Also the HV and DAQ are redeployed. The results show a fluctuation of $\sim 1 \%$.

\section{Measurement Results and Discussion}

Four WLS fibers of $5.4 \mathrm{~m}$ length are sampled from every spool ( $\sim 450 \mathrm{~m}$ in total) for measurement. Fibers are cleaned and ends are polished before testing. The range of the distance between the light source and the PMT is from 100 to $400 \mathrm{~cm}$. The final estimation on the attenuation length of the spool is the averaged value of four samples.

Figure 5(a) shows a typical testing data and the fitting results. It can be seen good linearity between the light intensity and the distance. Figure 5(b) shows the measured attenuation length for all tested spools so far. The mean value of the measurements is $312.2 \mathrm{~cm}$, and the standard deviation is $40.1 \mathrm{~cm}$. Only averaged values among four samples taken from one spool are shown. So the total number of tested samples is four times larger than the count shown in the plot. 


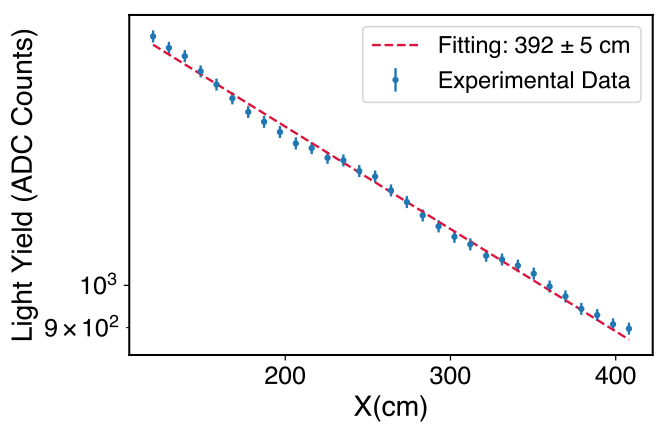

(a)

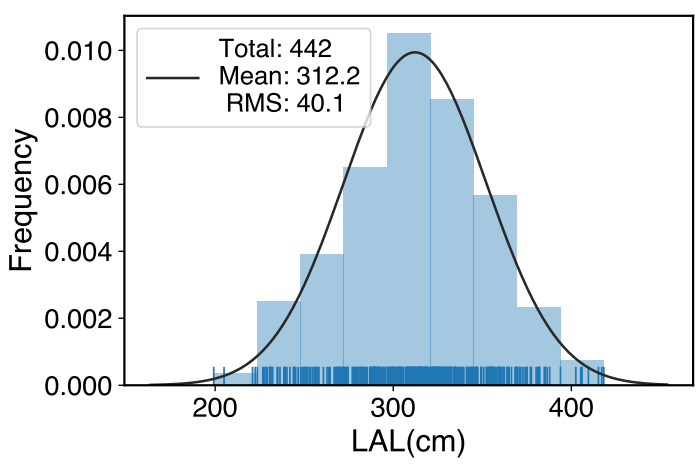

(b)

Figure 5: Measurement restults, (a) A typical data from one measurement (b) Histogram of attenuation length for all tested spools.

There are several issues that might have influence on the accuracy of the measurement.

1. Some aberrant data points with dramatically low values occasionally appear, which may result from the physical qualities of the WLS fiber other than the attenuation length, including impurity, diameter variation, microscopic damage of the cladding. We solve this problem by introducing a outlier detection procedure in the code to eliminate those abnormal data points;

2. It usually shows a much lower attenuation coefficient when the exitation point is too close to the fiber end[5], mainly due to the light propagation in the cladding. That's why we neglect the range of sub $100 \mathrm{~cm}$ in the measurement. Otherwise a double exponential fitting should be applied;

3. The attenuation length is wavelength-dependent[4]. One should be careful when comparing measured results by using photon sensors with a different spectral response (PMTs vs photodiodes, for example).

\section{Summary}

We report the measurement of attenuation length of large amount of WLS fibers for LHAASO electromagnetic detectors on a scale of hundreds of kilometers. The measurement is performed on a fiber testing system designed and built for the purpose. The system meets the needs of LHAASO project on WLS fiber quality control and has shown advantages in testing speed and accuracy compared to our former method.

\section{Acknowledgement}

This work is partly supported by NSFC No. 11605208 and the Knowledge Innovation Fund of IHEP, Beijing. 


\section{References}

[1] Z. Cao, For the LHAASO Collaboration, Status of LHAASO updates from ARGO-YBJ, Nucl. Instrum. Methods Phys. Res. A 742 (2014) 95-98

[2] H. He, For the LHAASO Collaboration, Design of the LHAASO detectors, Radiation Detection Technology and Methods (2018) 2:7

[3] Z. Zhang, et al., Study on the performance of electromagnetic particle detectors of LHAASO-KM2A, Nucl. Instrum. Methods Phys. Res. A 845 (2017) 429-433

[4] C. Alfieri, A.B. Cavalcante and C. Joram, A set-up to measure the optical attenuation length of scintillating fibres, LHCb Report (2015) LHCb-PUB-2015-011

[5] N. Amos, A. Bross and M. Lundin, Optical attenuation length measurements of scintillating fibers, Nucl. Instrum. Methods Phys. Res. A 297 (1990) 396-403 